\title{
Mechanics of gastroduodenal emptying
}

\section{A study of gastric and duodenal emptying with miniature balloons and intestinal glass electrodes}

\author{
J. RHODES, P. GOODALL, AND H. T. APSIMON \\ From the Departments of Medicine, Surgery, and Radiology, \\ The Royal Infirmary, Cardiff
}

EDITORIAL COMMENT Gastric emptying can occur with only small increases in antral pressure and even in the absence of any rise in pressure.

The $p \mathrm{H}$ of duodenal contents fluctuates widely. These fluctuations may be due to intermittent ejection of gastric acid contents. In this paper we report an investigation into the relationship between these fluctuations in duodenal $p \mathrm{H}$ and gastric emptying.

\section{METHODS}

SUBJECTS There were three normal people (nos. 2, 3, and 4) and four patients with duodenal ulcer (nos. 1, 5, 6, and 7).

THE TUBE A composite tube system was used (Fig. 1) which consisted of two intestinal electrodes (Cambridge Instrument Co. Ltd.), a reference lead from the calomel electrode, two air-filled balloons, and a terminal rubber bag which was filled with water. The two electrodes were $15 \mathrm{~cm}$. apart with a balloon $5 \mathrm{~cm}$. proximal to each. The individual tubes were joined at their ends by rubber collars. In two experiments (subjects 1 and 2) the tube was modified and a single antral balloon was used with three duodenal electrodes which were 5,12 , and $25 \mathrm{~cm}$. distal to the balloon.

PROCEDURE The tube was passed after the nasal cavity had been anaesthetized with cocaine. The position of the tube was at first adjusted under radiological control so that the proximal balloon was in the gastric antrum, the proximal electrode in the duodenal bulb, and the distal balloon and electrode near the duodeno-jejunal flexure (Fig. 2).

Antral and duodenal pressure patterns are different, and this difference was used throughout the experiments to locate the position of the balloon in relation to the pylorus. Thus, when the proximal balloon recorded a single rise in pressure three times each minute, it was inferred that the balloon was in the antrum, but when multiple rises in pressure, with an approximate frequency of 12 times every minute were recorded, it was inferred

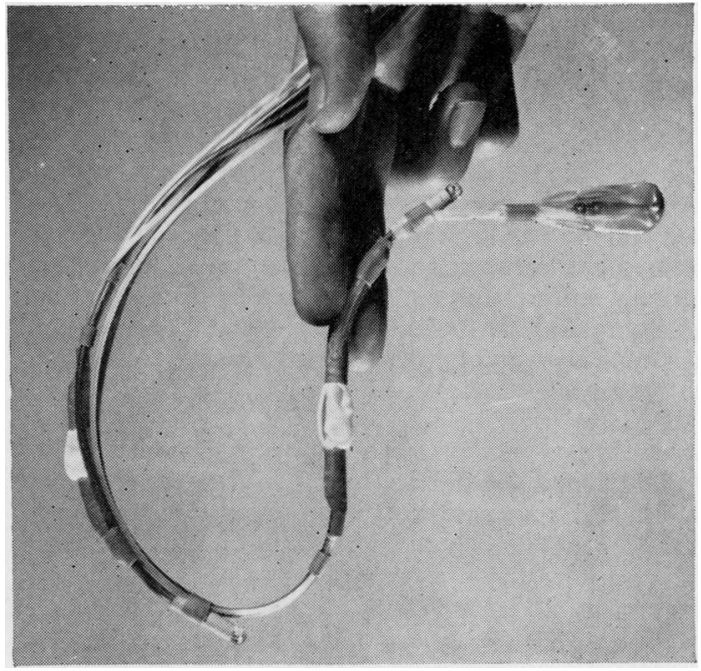

FIG. 1. The tube, with two glass electrodes, two small airfilled balloons, and a terminal weighted bag.

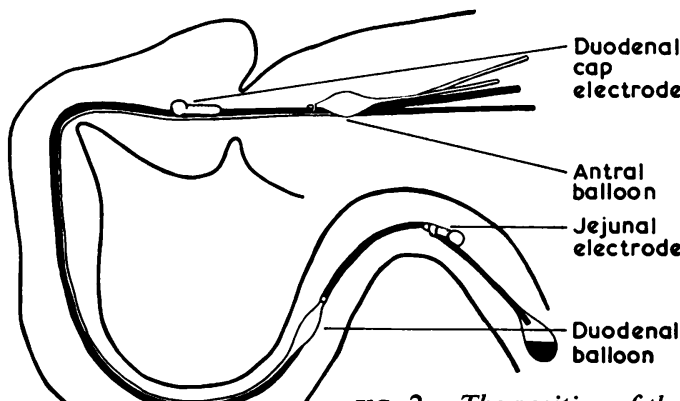

FIG. 2. The position of the tube in the stomach and duodenum. 
that the balloon was in the duodenum; it was then withdrawn a little.

Satisfactory records were not always obtained continuously because the tube moved out of position (Table I).

\section{TABLE I}

Patient No. Time (hr.) after Meal in which Satisfactory Records were Obtained

\begin{tabular}{ll}
\hline 1 & $\frac{1}{2}$ to 2 \\
2 & 0 to $1 \frac{1}{2}$ \\
3 & 1 to 7 \\
4 & 0 to $2 \frac{1}{2}$ \\
$5 a$ & 0 to 4 \\
$5 b$ & 0 to 9 \\
6 & 5 to 7 \\
7 & $2 \frac{1}{2}$ to 7
\end{tabular}

All subjects were given an ordinary meal of meat and vegetables. In subject 5 records were obtained after two meals.

RECORDS OF $p H$ AND PRESSURE The $p H$ and pressure were recorded simultaneously on an ultra-violet photographic recorder (S.E. Labs. Ltd./2000/12 U.V.) and continuous records obtained for between three and nine hours after the meal. The $\mathrm{pH}$ was recorded with $\mathrm{pH}$ meters (Model 23A Electronic Instruments Ltd.), and pressure with $1 \mathrm{ml}$. air-filled balloons which were connected to strain gauge manometers (Statham Transducers Inc. model P.23 Db). Each balloon was calibrated against a water manometer. For pressures between 0 and $60 \mathrm{~cm}$. of water, the relationship between pressure and the deflection of the recorder was linear and the balloon strain gauge system would respond to changes of pressure at a frequency greater than one cycle per second.

RADiOlogical OBSERVATIONS Subjects 1 and 2 were given barium sulphate suspension with their meals and the onward movement of radio-opaque medium was carefully observed on an image intensifier. A note was made of the coincidence of the following events: (a) waves of antral contraction with rises of antral pressure; $(b)$ the movement of gastric contents into the duodenal bulb with falls in the duodenal bulb $\mathrm{pH}$; (c) the movement of duodenal contents into the jejunum with falls of jejunal $p \mathrm{H}$.

ANALYSIS OF THE $p H$ RECORDS The following observations were made on the records.

The frequency of rises in antral pressure, and falls of bulb $\mathrm{pH}$ were measured and the relations between them were examined. Rises in antral pressure of less than $2 \mathrm{~cm}$. of water and falls in the duodenal bulb $\mathrm{pH}$ of less than $\mathbf{0 . 3}$ $p \mathrm{H}$ units were not included in the analysis.

The frequency of falls in the bulb and jejunal $p H$ were measured for three hours following the meal in subjects 1 , 4,5 , and 6.

The magnitude, as well as the frequency, of changes in the duodenal bulb $\mathrm{pH}$ and antral pressure were measured in subjects 1,4 , and 5 .

The records from normal and ulcer subjects were not compared.

\section{RESULTS}

In each subject, rises in antral pressure occurred about three times per minute and were usually accompanied by falls in the duodenal bulb $p H$. Rises in duodenal pressure occurred about once per minute and were accompanied by a fall in jejunal $p H$. A typical record of these events from one subject is shown in Figure 3.

ASSOCIATION OF CHANGES IN ANTRAL PRESSURE AND DUODENAL $p H$ The relation between rises in antral pressure and falls in the duodenal bulb $\mathrm{pH}$ for each patient is given in Table II. The averages of results
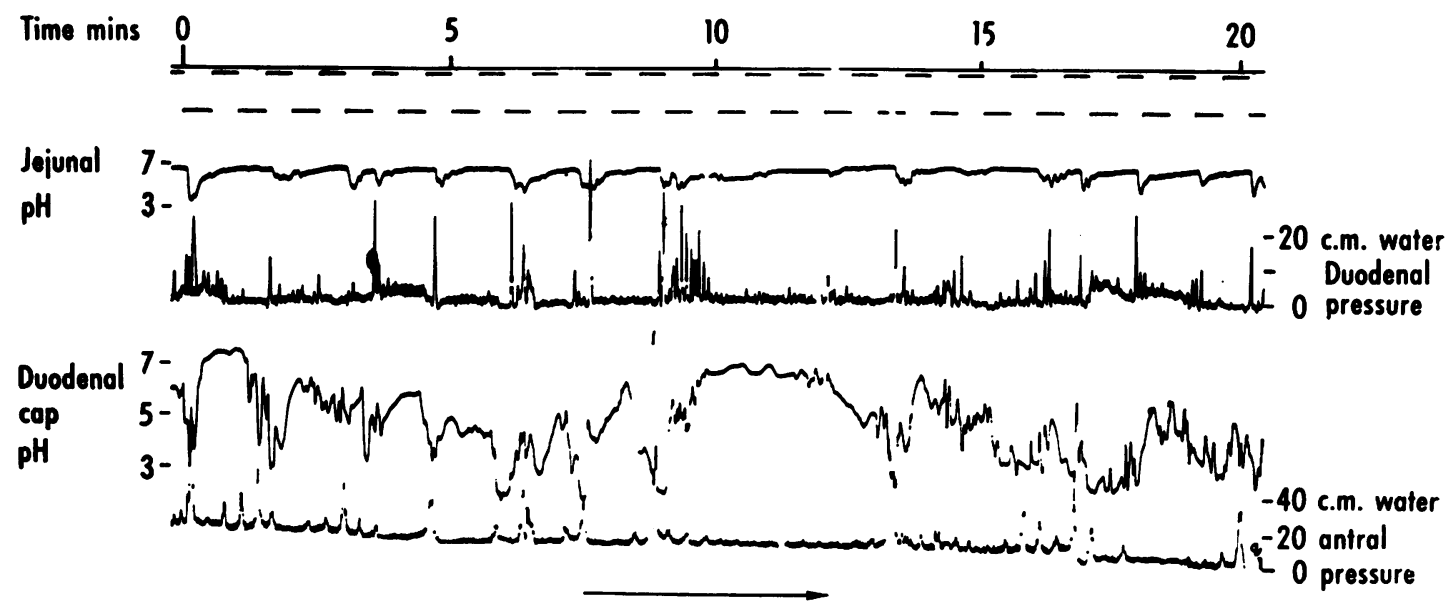

FIG. 3. Normal subject; simultaneous records of antral and duodenal pressure with duodenal bulb and jejunal pH. Rises in antral pressure are associated with a fall in duodenal $\mathrm{pH}$ and rises in duodenal pressure with $a$ fall in jejunal $\mathrm{pH}$. 
TABLE II

FREQUENCY OF RISES IN ANTRAL PRESSURE WITH DUODENAL ACIDIFICATION, RISES IN ANTRAL PRESSURE ALONE, AND DUODENAL ACIDIFICATION ALONE, IN EACH SUBJECT AFTER THE MEAL

Subject No. Time after Meal (hours)

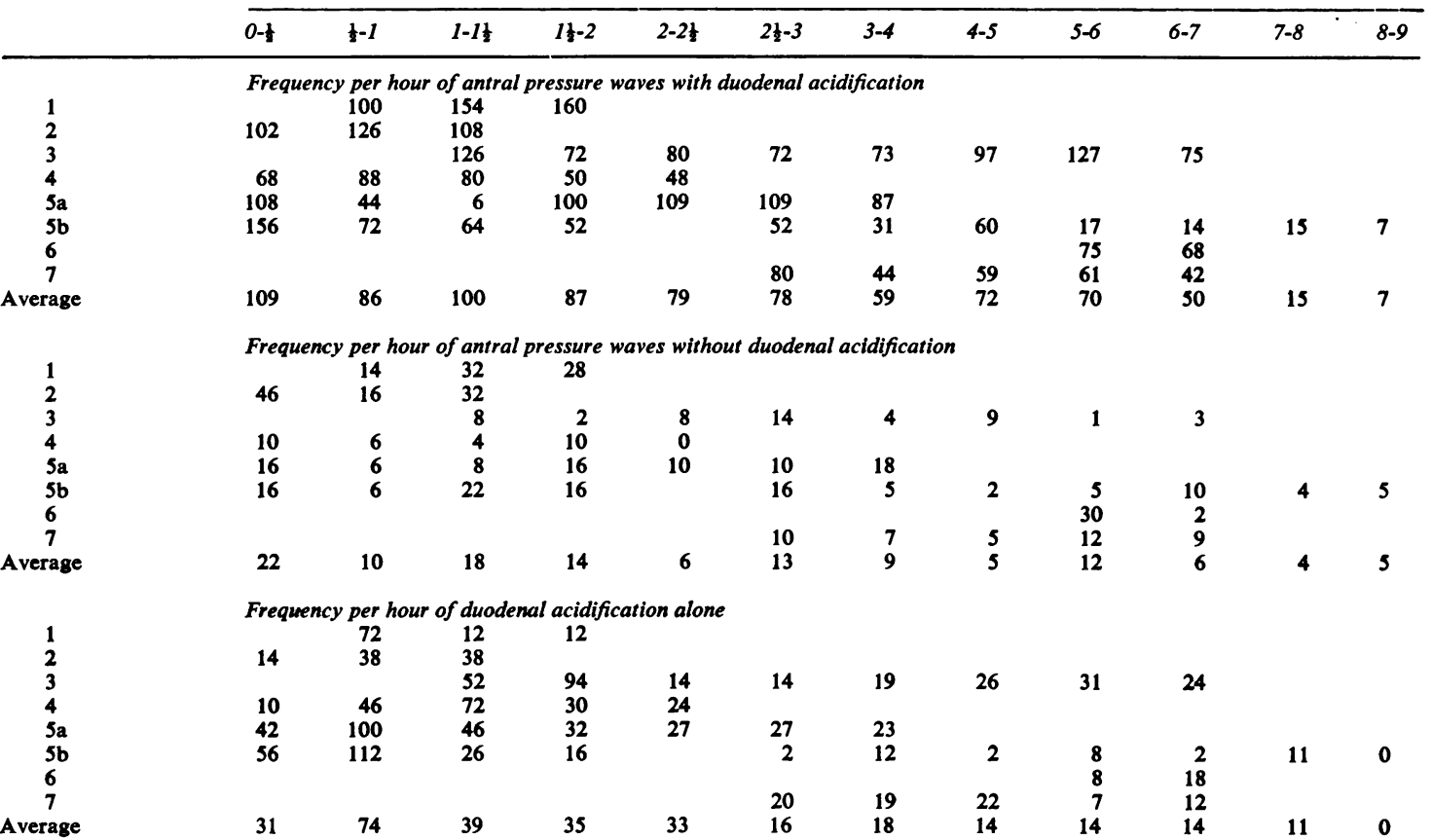

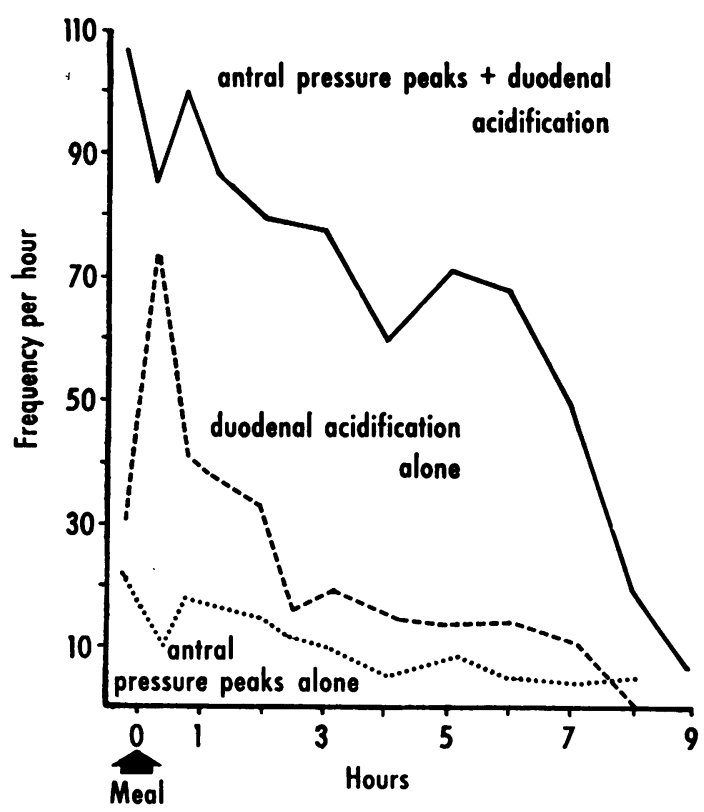

FIG. 4. The frequency of antral pressure peaks and duodenal acidification, alone and in combination after food. from all the patients, at different intervals after food are plotted in Figure 4. Rises in the antral pressure and duodenal acidification usually occurred together and were most frequent during the first one and a half hours after a meal. Only 5 to $17 \%$ of the rises in antral pressure were unaccompanied by duodenal acidification. During the first two and a half hours after a meal duodenal acidification often occurred in the absence of any detectable rise in the antral pressure (Fig. 5).

The mean falls in the duodenal bulb $\mathrm{pH}$ accompanying different rises in antral pressure are given in Table III. In general it was found that large rises in pressure were associated with large falls in the bulb $p \mathrm{H}$. However, the relationship between the two events is by no means precise, and wide variations occur.

ASSOCIATION OF CHANGES IN THE DUODENAL PRESSURE AND JEJUNAL $p_{H}$ Recordings from the distal balloon and electrode were also studied. Unlike pressure rises recorded in the antrum, those in the duodenum were of a complex nature (Fig. 3). Rises in duodenal pressure occurred as a series of simple spikes, at a rate of 12 per minute appearing in groups each last- 


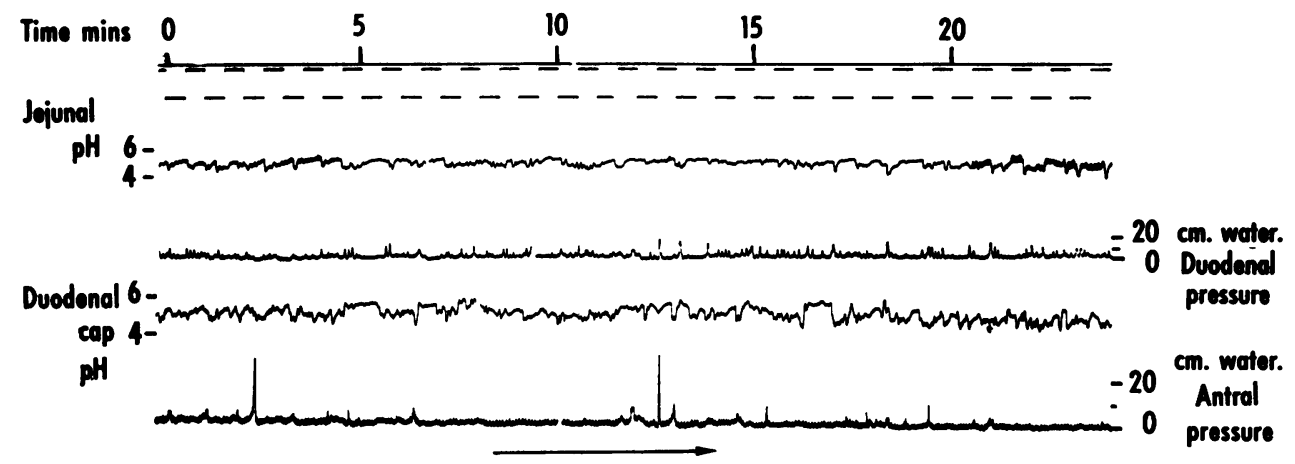

FIG. 5. Normal subject; simultaneous records of antral and duodenal pressure with duodenal bulb and jejunal $\mathrm{p} H$ with few antral waves and irregular gastric emptying 30 minutes after a meal. Falls in the duodenal bulb $\mathrm{pH}$ often occur without a rise in the antral pressure.

\section{TABLE III}

MAGNITUDE OF CHANGES IN $p H$ AND ANTRAL PRESSURE IN PATIENTS 1,4 , AND 5

Patient No. Rise in Antral Pressure $\left(\mathrm{cm}^{\mathrm{H}} \mathrm{H}_{2} \mathrm{O}\right)$

\begin{tabular}{rrrr}
\hline & \multicolumn{2}{c}{$10-20$} & \multicolumn{1}{c}{$<10$} \\
1 & $\Delta p H^{1} 3.05(11)^{2}$ & $p H 3.04(15)$ & $p H 1.22(40)$ \\
4 & $2.46(33)$ & $2.19(20)$ & $1.25(27)$ \\
5 & $1.85(20)$ & $1.8(20)$ & $0.95(20)$
\end{tabular}

${ }^{1} \Delta p \mathrm{H}$ is the mean fall in $p \mathrm{H}$ associated with a group of rises in antral pressure of similar magnitude.

${ }^{2}$ The number of waves counted is given in brackets.

ing about 30 seconds. These simple spikes occurred on a rise in the base line pressure and were associated with a fall in the $p \mathrm{H}$. In addition a few complex duodenal waves were seen which lasted for several minutes. These always occurred at least seven hours after food and followed a sudden large fall in the duodenal $p \mathrm{H}$; the duodenal contractions then continued for between four and eight minutes after the duodenal $p \mathrm{H}$ had returned to normal.

The jejunum was acidified less often than the duodenal bulb. There was a fall in the jejunal $p \mathrm{H}$ every 50 seconds in the first hour after food, but every 70 to 80 seconds during the following two hours. There was a fall in the duodenal $p \mathrm{H}$ every 20 to 25 seconds. The approximate ratio between duodenal bulb and jejunal acidification was 2 to 1 in the first hour and 3 to 1 later (Fig. 6). Falls in the jejunal $p \mathrm{H}$ were always preceded by a rise in duodenal pressure.

RADIOLOGICAL OBSERVATIONS ON GASTRIC EMPTYING It was found during screening that propulsive contractions which moved across the gastric antrum were associated with a rise in the antral pressure. Gastric contents could sometimes be seen to move onwards into the duodenal bulb as the antral con- traction approached the pylorus. This was associated with a fall in the duodenal $p \mathrm{H}$. Many of the smaller rises in the antral pressure were not associated with visible movement of gastric contents into the bulb, but were associated with duodenal acidification, indicating the onward passage of gastric contents, which could not be detected radiologically. Rises in the duodenal pressure and jejunal acidification were associated with the passage of a bolus around the duodenal loop.

The sequence of events during gastric emptying was that propulsive contractions arose near the incisura angularis and progressed smoothly towards the pylorus. Several antral waves caused the duodenal bulb to fill. The bulb then contracted and its contents were propelled around the duodenal loop. Multiple contractions were then seen in the second part of the duodenum and were associated with the reflux of contents from the second part of the duodenum into the duodenal bulb.

The $p \mathrm{H}$ changes in the duodenal bulb, the third part of the duodenum and jejunum, associated with this sequence can be seen in Figure 6. After the bulb has emptied, as shown by the fall in the $p \mathrm{H}$ of the third part of the duodenum, there is an immediate rise in the $p \mathrm{H}$ of the contents of the bulb. This rise of $p \mathrm{H}$ was associated with the visible reflux of contents from the duodenal loop into the bulb. A more gradual rise of $p \mathrm{H}$ then occurs between the successive falls; at these times the bulb filled.

\section{DISCUSSION}

Simultaneous measurement of the antral pressure and the duodenal bulb $p \mathrm{H}$ has made it possible to examine the association between them. The cause of fluctuations in the duodenal bulb $p \mathrm{H}$ has been studied by Besançon, Chérigié, Hébert, and Debray 


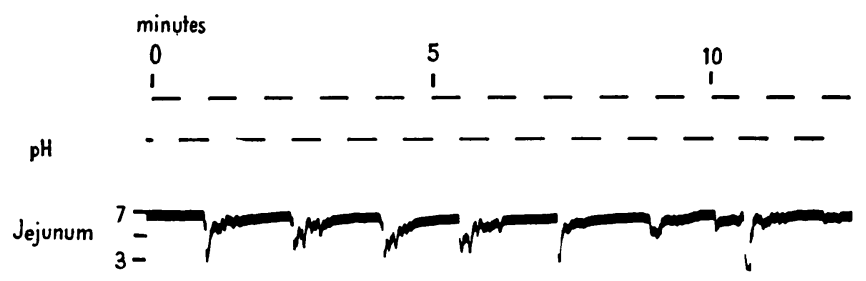

3rd part
duodenum $3-$

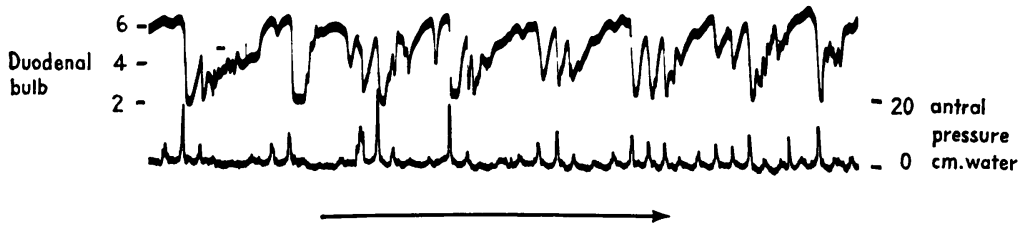

FlG. 6. Duodenal ulcer patient: simultaneous records of pressure in the antrum with $\mathrm{pH}$ in the duodenal bulb, third part of the duodenum and jejunum. There is a fall in the duodenal bulb $\mathrm{pH}$ about three times each minute and in the jejunal $\mathrm{pH}$ about once each minute.
(1961). They used cineradiography while recording the duodenal bulb $p \mathrm{H}$, and found that the fluctuations were associated with passage of food through the pylorus and with reflux from the second part of the duodenum. These observations could only be made for short periods, whereas with an intestinal glass electrode one can record changes of duodenal $p \mathrm{H}$ due to gastric emptying for long periods.

We have found that rises in the antral pressure are usually associated with a fall in the duodenal bulb $p \mathrm{H}$. Rises in the antral pressure which occur about three times each minute have previously been classified according to their size. Type I waves, which are smaller than $10 \mathrm{~cm}$. of water, have been named 'mixing' waves, while those greater than $10 \mathrm{~cm}$. of water (type 11) were thought to be propulsive and to cause gastric emptying (Code, Hightower, and Morlock, 1952). Most observations relating antral pressure to gastric emptying in man have relied on $x$-ray fluoroscopy. Our observations show that the passage of small quantities of gastric contents cannot always be detected by this method. With an intestinal glass electrode, however, it has been shown that even small increases in antral pressure may cause gastric emptying. Moreover, our findings indicate that gastric emptying can occur in the absence of a recordable rise in the antral pressure, either because some antral contractions do not give a recordable change in pressure (Connell and Rowlands, 1960), or because a distended stomach will empty without antral propulsive waves.

Most duodenal pressure waves consist of a series of simple waves superimposed on a rise in the base line pressure (Fig. 3). These have been classified as type III waves (Templeton and Lawson, 1931; Code et al., 1952): they are primarily propulsive in character and associated with jejunal acidification. During the fasting state a few prolonged type III waves occurred. These were not associated with the propulsion of acid duodenal contents and their significance is not fully understood. Duodenal contractions appear to be initiated by gastric contents entering the duodenum; the stimulus to contraction of the bulb may be distension, acidification of its contents, or both. Brink, Schlegel, and Code (1965) have shown in dogs that instillation of acid into the duodenum stimulates duodenal contractions. Our studies have shown that the same occurs in man and that the pressure pattern produced by acid differs from that produced by a similar volume of normal saline or by an inert bolus.

The reflux of contents from the second part of the duodenum into the bulb was accompanied by neutralization of the contents of the bulb. This reflux must be associated with a difference in pressure at the two sites, which develops after the bulb has contracted. Reflux of contents from the second part of the duodenum into the bulb is often seen during a barium meal examination. The same phenomenon is also seen after contrast has been injected through a $T$ tube into the common bile duct; when the opaque medium enters the duodenum it is often carried into the bulb before being propelled around the duodenum.

With two balloons and two electrodes it was possible to examine the sequence of events during gastroduodenal emptying. Two or three antral contractions filled the duodenal bulb which then contracted and propelled its contents around the duodenum (Fig. 6). 


\section{SUMMARY}

The relationship between fluctuations in duodenal pH and intraluminal pressure in the stomach and duodenum was investigated. Falls in the duodenal bulb $p \mathrm{H}$ were usually accompanied by a rise in the antral pressure, but during the first two and a half hours after a meal there was often a fall in $p \mathrm{H}$ without a rise in the antral pressure. During stomach emptying, after several antral contractions the duodenal bulb was filled and then contracted, propelling its contents around the duodenum. Reflux of alkaline contents from the second part of the duodenum into the bulb was partly responsible for the fluctuations in $p \mathrm{H}$.

We are grateful to Professor H. Scarborough, Dr. A. H. James, and Professor A. P. M. Forrest for their help, and to Mr. R. Marshall for preparing the illustrations. The work was carried out while J. Rhodes was the Cardiff Royal Infirmary Research Fellow in Medicine.

\section{REFERENCES}

Besançon, F., Chérigié, E., Hébert, F., and Debray, C. (1961). La 'cinéradiométrie'. Synchronisation de la radiocinématographie avec l'electromanométrie et la pHmétrie: lecture directe des mesures endocavitaires sur les films de l'oesophage du bulbe duodénal, de l'intestin grêle et du cholédoque. C.R. Soc. Biol. (Paris), 155, (1) 291-293.

Brink, B. M., Schlegel, J. F., and Code, C. F. (1965). The pressure profile of the gastroduodenal junction zone in dogs. Gut, 6, 163-171.

Code, C. F., Hightower, N. C., Jr., and Morlock, C. G. (1952). Motility of the alimentary canal in man: review of recent studies. Amer. J. Med., 13, 328-351.

Connell A. M., and Rowlands, E. N. (1960). Wireless telemetering from the digestive tract, Gut, 1, 266-272.

Kay, A. W. (1953). Effect of large doses of histamine on gastric secretion of $\mathrm{HCl}$. An augmented histamine test. Brit. med. J., 2, 77-80.

Templeton R. D., and Lawson, H. (1931). Studies on the motor activity of the large intestine: normal motility in the dog, recorded by the tandem balloon method. Amer. J. Physiol., 96, 667-676. 